\title{
The history of IAU Symposia
}

\author{
Areg M. Mickaelian \\ Byurakan Astrophysical Observatory (BAO), \\ Byurakan 0213, Aragatzotn Province, Armenia \\ email: aregmick@yahoo.com
}

\begin{abstract}
The history of the International Astronomical Union (IAU) meetings goes back to 1922 when the first IAU General Assembly (GA) was held in Rome, Italy, following the IAU creation in 1919. However, until 1953, no individual symposia were organized and the GAs were the only official gatherings for astronomers. All together, eight IAU GAs were held during 1922-1952. The IAU Symposium 1 was held in 1953 in Groningen, Netherlands. Starting with 1955, several IAU symposia were regularly held in different places, and since 1959, the IAU also began to organize colloquia to discuss relatively smaller topics. Twenty IAU colloquia numbered as I-XX were held in the period 1959-1971, and another series of IAU colloquia was organized in 1968-2005, numbered as Nos. 1-200. At present IAU symposia are the only official scientific meetings, nine of them being organized every year. IAU S349 "Under One Sky: the IAU Centenary Symposium", held in Vienna during the IAU GA XXX, was the last one by number in 2018. Thus, the IAU has a 65 -year history of symposia and all together 348 such meetings have been held, on average 5-6 annually. At present most of the IAU symposia during the years of GA are being organized in the framework of the GA, there being typically six symposia during each GA. All together, 31 IAU GA have been organized during the years 1922-2018, including 30 regular ones and one Extraordinary GA (1973 in Warsaw, Poland), typically once every three years. Since 1974, the IAU has also organized regional meetings in Europe, Asia and Pacific (APRIM), Latin America (LARIM), and the Middle East and Africa (MEARIM). The European ones were discontinued in 1990 after the creation of the European Astronomical Society (EAS) and the organization of the yearly JENAM/EWASS. The 348 IAU symposia have been organized in 43 countries. We give the statistics of all IAU symposia by year of organization, by various topics of astronomy and astrophysics, and by host countries and cities.
\end{abstract}

Keywords. IAU, history, meetings, symposia

\section{Introduction}

The International Astronomical Union (IAU) meetings started in 1922 when the first IAU General Assembly (GA) was held in Rome, Italy, following the IAU creation in 1919. However, until 1953, no individual symposia were organized and the GA was the only official gathering for astronomers. All together, eight IAU GA were held during the period 1922-1952.

The IAU Symposium no. 1 on "Co-ordination of Galactic Research" was held during June 17-22, 1953 in Groningen, Netherlands, followed by another one (IAU Symposium no, 2: "Gas Dynamics of Cosmic Clouds") on July 6-11 in Cambridge, UK. Starting with 1955, several IAU symposia were regularly held in different places, and since 1959, the IAU also began to organize colloquia to discuss relatively smaller topics. Twenty IAU colloquia, numbered as I-XX, were held in the period 1959-1972, and another series of IAU colloquia was organized in 1968-2005, numbered as Nos. 1-200. At present IAU symposia are the only official scientific meetings, nine of them being organized every year. IAU S349 "Under One Sky: the IAU Centenary Symposium", held in Vienna during the 


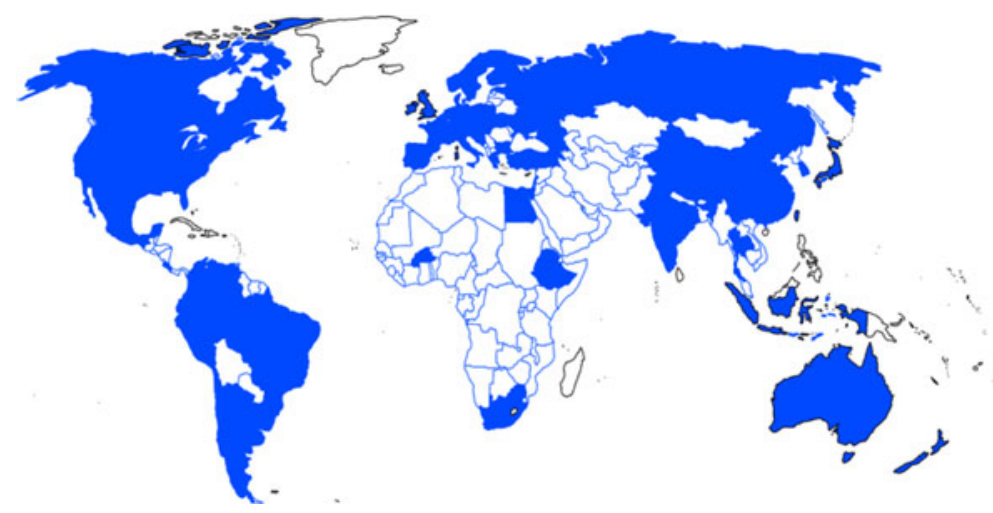

Figure 1. The world map showing 49 countries which have hosted IAU symposia.

IAU GA XXX, was the last one by number in 2018. Thus, IAU has a 65 -year history of symposia, and all together 348 such meetings have been held, on average five annually. One symposium, IAU S326 was cancelled in 2016.

\section{IAU symposia}

At present most of the IAU symposia during the years of a GA are being organized in the framework of the GA. There are typically six symposia during each GA. They were as follows:

- IAU GA XXII: IAU GA 1994, Hague, Netherlands - 6 symposia

- IAU GA XXIII: 1997, Kyoto, Japan - 6 symposia

- IAU GA XXIV: 2000, Manchester, UK - 5 symposia

- IAU GA XXV: 2003, Sydney, Australia - 6 symposia

- IAU GA XXVI: 2006, Prague, Czech Republic - 6 symposia

- IAU GA XXVII: 2009, Rio de Janeiro, Brazil - 6 symposia

- IAU GA XXVIII: 2012, Beijing, China - 8 symposia

- IAU GA XXIX: 2015, Honolulu, Hawaii, USA - 6 symposia

- IAU GA XXX: 2018, Vienna, Austria - 7 symposia

All together, 31 IAU GA have been organized during the years 1922-2018, including 30 regular ones and one Extraordinary GA (1973 in Warsaw, Poland). Typically the GA are held once every three years.

The 348 meetings have been organized in 49 countries; most often in the USA (50), France (25), Italy, UK, and Australia (each 21), China Nanjing (20), Germany (15), Japan, Netherlands, and Brazil (each 14), Austria (11), Switzerland (10), Spain and Canada (each 9), Russia (8), Argentina, India, Czech Republic, Poland, and Sweden (6), Armenia (5). All together, 192 cities and towns have been hosts for the IAU symposia, most often Beijing (12), Paris (12), Vienna (10), Sydney (9), Rio de Janeiro (8), Kyoto (7), Prague (7), Geneva (6), Honolulu, HI (6), The Hague (6), Byurakan (5), Leningrad / St.Petersburg (5), Manchester (5).

The distribution of the IAU symposia by countries is given in Figure 1. The distribution by continents is as follows:

- 187 Europe (28 countries)

- 64 North America (4 countries)

- 42 Asia (5 countries)

- 26 South America (7 countries)

- 23 Australia and Oceania (2 countries)

- 6 Africa (3 countries) 

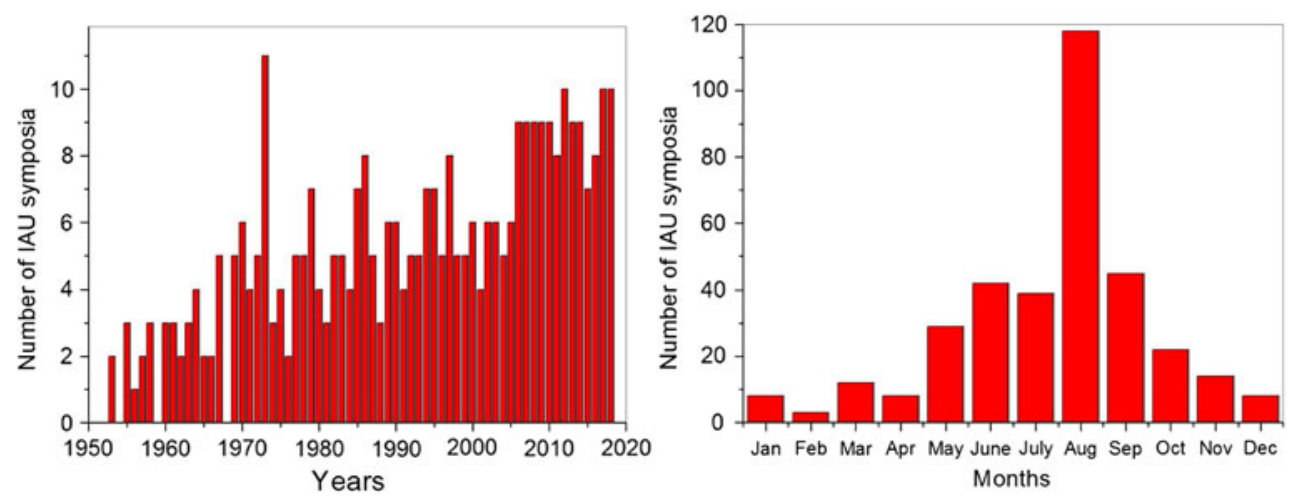

Figure 2. Distribution of the number of IAU symposia by year (left) and by month (right).

IAU symposia started in Europe (Groningen, Netherlands) in 1953. The first symposium outside of Europe (North America) was held in 1957 in Cambridge (MA, USA). Then the geography of IAU symposia was expanded to Australia (1963, Canberra), Asia (1971, Morioka, Japan, and also in 1966 in Byurakan, Armenia (then part of the USSR), South America (1971, Buenos Aires, Argentina) and Africa (1998, Cape Town, South Africa).

The distribution of the IAU symposia by years and by months is given in Figure 2 . An increase in numbers is clearly seen in case of the distribution by years. However interestingly, the maximum number of IAU symposia during a year (11) was held in 1973, not in the recent years. Since 2006, the average number of annual IAU symposia is nine, and 10 symposia were held in each of the last 2 years 2017 and 2018).

Regarding the distribution by months, most often (118 times) IAU symposia have been held in August, mainly connected to the IAU GA. September (45), June (42), July (39), May (29) and October (22) also have big numbers of symposia. Only three symposia have been held in February.

The 348 IAU symposia have been held on various topics of astronomy and astrophysics (more than 40 subjects by our classification). The subjects most often found were:

- various matters related to stars (37)

- various astrophysical processes (28)

- galaxies (27)

- $\operatorname{cosmology}(17)$

- Sun (17, including solar activity 7$)$

- Solar System (16)

- star formation and evolution (15)

- instrumentation and methods (14)

- multiwavelength astronomy ( $\gamma$-ray, X-ray, UV, IR and radio) (13)

- AGN (10)

- exoplanets and SETI (9)

- Our Galaxy (9)

- planetary nebulae (9)

- astrochemistry (8)

- binary stars (8)

- interstellar medium (8)

- astrometry (7)

- Earth (7)

- fundamental astronomy (6)

- magnetic fields (6) 


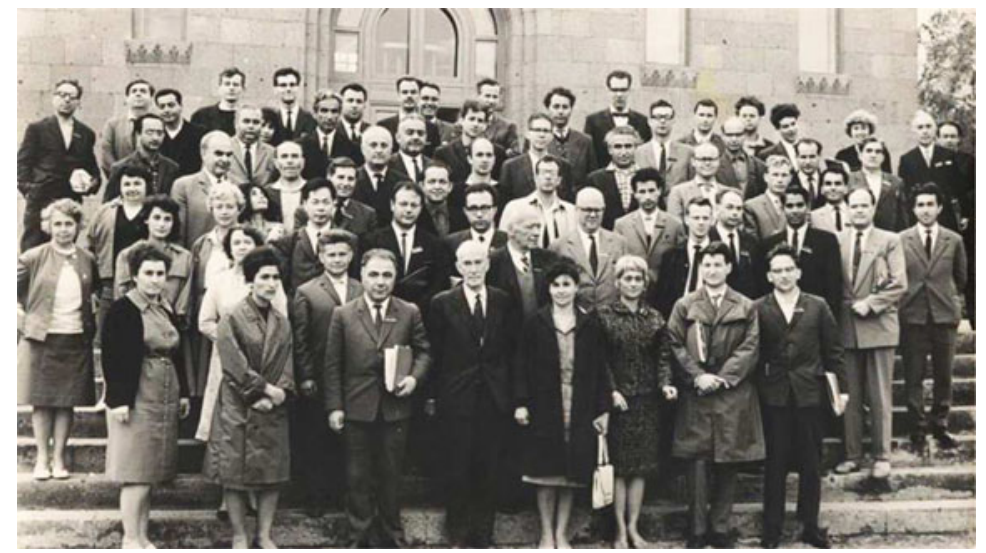

Figure 3. Group photo of the IAU Symposium No. 29: Non-Stable Phenomena in Galaxies, held on 4-12 May 1966 in Byurakan, Armenia. Viktor Ambartsumian and Jan Oort on the front row.

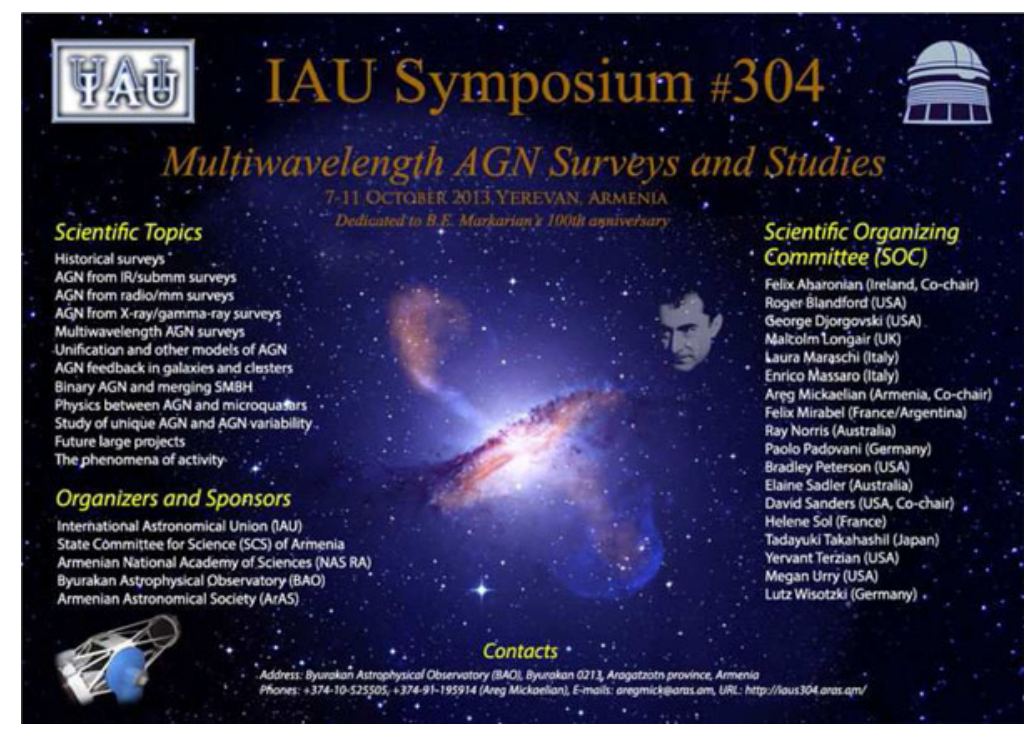

Figure 4. The official poster of the IAU Symposium No. 304: Multiwavelength AGN Surveys and Studies, held 7-11 October 2013 in Yerevan, Armenia.

- star clusters (6)

- Magelanic Clouds (5)

- neutron stars and pulsars (5)

- Wolf-Rayet stars (5)

- Supernovae (4)

- astronomical surveys (4)

IAU symposia played and play an important role in astronomy and may be considered as the highest level meetings. At present the typical number of participants at an IAU symposium exceeds 200 .

We give in Figure 3 the group photo of the IAU Symposium \#29 (1966) and in Figure 4, the official poster of the IAU Symposium \#304 (2013), both held in Byurakan, Armenia. 


\section{IAU regional meetings}

Since 1974, the IAU has also organized regional meetings in Europe, Asia and Pacific (APRIM, Asia-Pacific Regional IAU Meeting), Latin America (LARIM, Latin-American Regional IAU Meeting), and the Middle East and Africa (MEARIM, Middle East and African Regional IAU Meeting). The European ones were discontinued in 1990 after the creation of the European Astronomical Society (EAS) and organization of the yearly JENAMs (at present EWASS - European Week of Astronomy and Space Science). The three others, APRIM (since 1978), LARIM (since 1978) and MEARIM (since 2008) are being organized every two or three years. All together 44 regional meetings have been organized, including:

- 12 IAU European Regional Meetings

- 13 APRIM

- 15 LARIM

- 4 MEARIM

\section{Discussion}

LATTIS: Were the Symposia in 1973, which was the peak year, historical in nature?

MickAelian: No, significant historical Symposia came later.

\section{References}

Information related to IAU Symposia and Colloquia: see www.iau.org/publications/iau 